\title{
高振动激发态的 $\boldsymbol{V}-\boldsymbol{V}$ 传能研究 - II . $\mathrm{CO}(v)$ 向 $\mathrm{H}_{2} \mathrm{O}$ 的振动传能
}

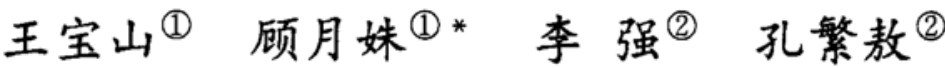

(1)山东大学化学系, 济南 250100; (2)中国科学院化学研究所分子反应动力学国家重点实验室, 北京 100080. * 联系人)

摘要 利用时间分辨 Fourier 红外发射光谱仪 (TR FTIR), 对高振动激发的 $\mathrm{CO}(v)$ 向 $\mathrm{H}_{2} \mathrm{O}$ 的 传能反应进行了研究. 传能给体分子 $\mathrm{CO}(v)$ 由 $193 \mathrm{~nm}$ 激光光解 $\mathrm{CHBr}_{3}$ 和 $\mathrm{O}_{2}$ 后的次级化学 反应产生. $\mathrm{CO}(v \rightarrow v-1)$ 的红外发射由 TR FTIR 跟踪检测. 实验中没有观察到 $\mathrm{H}_{2} \mathrm{O}$ 的振动 激发. 利用光谱拟合方法, 得到每一延时 $\mathrm{CO}(v=1 \sim 8)$ 的振动布居, 进而求出 $\mathrm{CO}$ 各个振动激 发态的相对布居随时间的演化关系。在单量子跃迁模型的基础上,利用微分法得到室温下 $\mathrm{CO}(v=1 \sim 8)$ 向 $\mathrm{H}_{2} \mathrm{O}$ 的传能速率常数. 至少有 2 个原因导致 $\mathrm{CO}(v)$ 向 $\mathrm{H}_{2} \mathrm{O}$ 弛豫的异常快的 速率: $\mathrm{H}_{2} \mathrm{O}$ 的转动对振动能隙的补偿; $\mathrm{CO}$ 和 $\mathrm{H}_{2} \mathrm{O}$ 分子间形成一定寿命的络合物. 结合 $\mathrm{SSH}$ 理论和 $a b$ initio 计算, 对 $\mathrm{CO}$ 向 $\mathrm{H}_{2} \mathrm{O}$ 的传能机理进行了解释.

\section{关键词 时间分辣 红外光谱 高振动潄发态 振动传能}

在烃类火焰中存在着大量的 $\mathrm{CO}$ 高振动激发态及 $\mathrm{H}_{2} \mathrm{O}$ 蒸气分子. 研究高振动激发态 $\mathrm{CO}$ 与 $\mathrm{H}_{2} \mathrm{O}$ 的传能, 无疑会对燃烧化学中这一重要的传能过程有进一步的认识. Stephenson 和 Mosbury ${ }^{[1]}$ 利用 LIF 技术得到了 $\mathrm{CO}(v=1)$ 与 $\mathrm{H}_{2} \mathrm{O}$ 的传能速率常数为 $2 \times 10^{-13} \mathrm{~cm}^{3}$. molecule ${ }^{-1} \cdot \mathrm{s}^{-1}$. 至今未见 $\mathrm{CO}$ 的高振动激发态向 $\mathrm{H}_{2} \mathrm{O}$ 传能的报道.

$\mathrm{H}_{2} \mathrm{O}$ 是一个比较特殊的传能受体分子. 即使有少量 $\mathrm{H}_{2} \mathrm{O}$ 存在, 也会使传能大大加 快 ${ }^{[2,3]} . \mathrm{H}_{2} \mathrm{O}$ 有两个氢原子, 转动惯量小, $\mathrm{V}-\mathrm{R}$ 传能较快. $\mathrm{H}_{2} \mathrm{O}$ 还容易和其他分子形成氢键络 合物, 导致传能速率加快 ${ }^{[4,5]}$. 因此研究 $\mathrm{CO}(v)$ 与 $\mathrm{H}_{2} \mathrm{O}$ 的传能反应, 可以加深对含氢体系传 能本质的了解.

我们在前文报道了 $\mathrm{CO}(v=1 \sim 8)$ 与 $\mathrm{CO}_{2}$ 的传能 ${ }^{[6]}$. 本文则首次获得了 $\mathrm{CO}(v=1 \sim 8)$ 向 $\mathrm{H}_{2} \mathrm{O}$ 的传能速率常数, 认为 $\mathrm{CO}(v) \rightarrow \mathrm{H}_{2} \mathrm{O}\left(\nu_{2}\right)$ 是最可能的传能通道, 并探讨了 $\mathrm{CO}$ 与 $\mathrm{H}_{2} \mathrm{O}$ 碰 撞时形成氢键络合物的可能性.

\section{1 实验方法}

实验装置及原理见文献 $[6]$. 用 $\mathrm{O}_{2}$ 通过气体流量计掠过 $\mathrm{CHBr}_{3}$ 液面直接进样; $\mathrm{H}_{2} \mathrm{O}$ (蒸 馏水) 蒸气由另一路管道由针阀控制通入光解反应室. 控制机械原的抽速, 既能使 $\mathrm{H}_{2} \mathrm{O}$ 的压 力保持恒定, 又能使光解反应区的物种在下次激光脉冲到来之前及时更新.

\section{2 结果和分析}

(1) 光谱分辨率设置为 $16 \mathrm{~cm}^{-1}, \mathrm{CHBr}_{3}, \mathrm{O}_{2}$ 和 $\mathrm{H}_{2} \mathrm{O}$ 的分压分别为 5,15 和 $50 \mathrm{~Pa}$. 依次得 到在激光脉冲后第 $10,60,110, \cdots 860 \mu \mathrm{s}$ 记录的 18 张红外发射光谱, 见图 1. 为了提高信噪 比, 实验数据重复累加 20 次. 图中 $1800 \sim 2200 \mathrm{~cm}^{-1}$ 为 $\mathrm{CO}$ 的 $(v \rightarrow v-1)$ 红外发射信号. 未 见 $\mathrm{H}_{2} \mathrm{O}$ 的 $\nu_{1}\left(3652 \mathrm{~cm}^{-1}\right)$ 或 $\nu_{3}\left(3756 \mathrm{~cm}^{-1}\right)$ 信号. 由于 InSb 探头在 $1800 \mathrm{~cm}^{-1}$ 截止, 不能观 
察 $\mathrm{H}_{2} \mathrm{O}$ 的弯曲振动模 $\nu_{2}\left(1596 \mathrm{~cm}^{-1}\right)$. 随着时间的延长, $\mathrm{CO}$ 光谱总强度逐渐下降, 而且向高 波数方向偏移,谱区逐渐变窄,这反映了 $\mathrm{CO}$ 高振动激发态被 $\mathrm{H}_{2} \mathrm{O}$ 弛豫的一个过程.

(2) 用最小二乘法模拟红外发射谱. 图 2 所示为 $260 \mu \mathrm{s}$ 时 $\mathrm{CO}(v \rightarrow v-1)$ 的光谱及其模 拟. 在本实验条件下, $\mathrm{CO}$ 的转动已充分驰豫至平衡, 转动温度取为 $300 \mathrm{~K}$. 为了能清楚地看 出各振动态的相对贡献, 图中画出了 $v=2,4,9$ 的振动带. 拟合光谱与实验光谱符合很好.

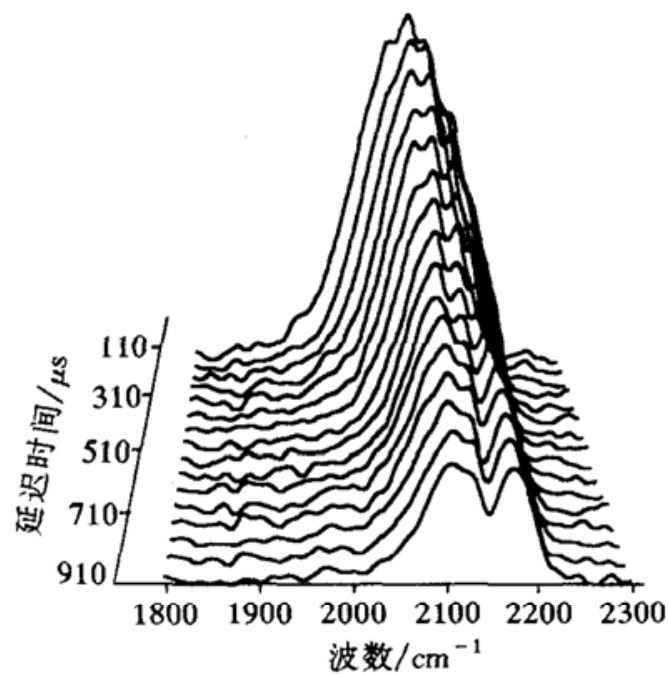

图 $1193 \mathrm{~nm}$ 光解 $\mathrm{CHBr}_{3}+\mathrm{O}_{2}+\mathrm{H}_{2} \mathrm{O}$ 体系 $\mathrm{CO}(v)$ 的时间分辨红外发射光谱

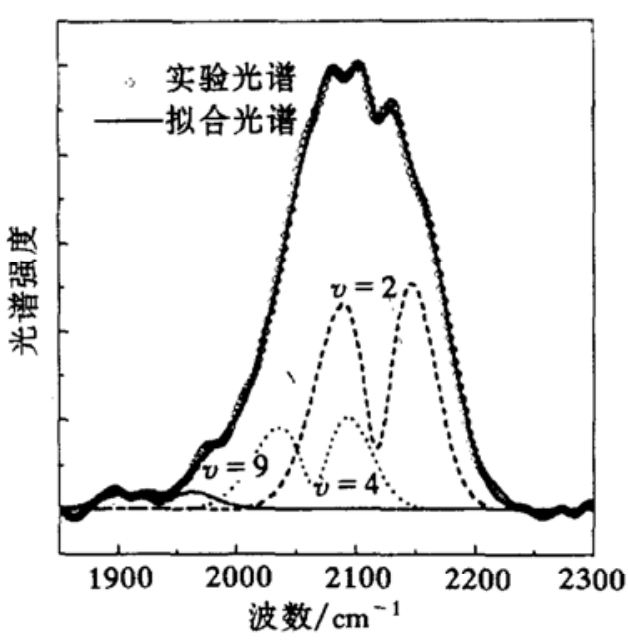

图 $2260 \mu \mathrm{s}$ 时 $\mathrm{CO}$ 发射光谱的拟合与实验光谱的 比较

图中给出了 $v=2,4,9$ 三个振动光谱带

(3) 通过模拟得到 $\mathrm{CO}(v)$ 各振动态在不同时刻的相对布居, 见图 3. 由于 $v>6$ 的高振动 态布居很小, 拟合误差较大, 图中没有绘出. 可以看 出, $\mathrm{CO}$ 高振动态向低振动态弛豫较快, 高振动态 $(v \geqslant 3)$ 布居迅速驰豫减小, 造成低振动态布居逐渐积 累. 对 $v=2$ 振动态来说, 在初始时刻即已形成对 $v=1$ 的布居反转. 自激光脉冲 $250 \mu \mathrm{s}$ 后逐渐下降. 由于高振动态比低振动态驰豫快, 造成了 $\mathrm{CO}(v=1)$ 的布居在 $600 \mu \mathrm{s}$ 内一直增加, 然后才缓慢下降.

(4) 根据文献 [6]的微分法对 $\mathrm{CO}-\mathrm{H}_{2} \mathrm{O}$ 的传能过 程进行了处理。在本实验中, 虽然光谱的分辨率较低 $\left(16 \mathrm{~cm}^{-1}\right)$, 但得到的光谱比较平滑, 信噪比很好, 拟 合布居起伏较小,适于用微分法处理. 为了尽量避免 $\mathrm{CO}(v)$ 的生成及自发辐射过程等因素的影响 ${ }^{[7]}$, 我们 从激光辐照后延时 $210 \mu \mathrm{s}$ 开始计算, 得到了 $\mathrm{CO}(v=$ $1 \sim 8)$ 向 $\mathrm{H}_{2} \mathrm{O}$ 传能的速率常数, 列于表 1 . 其中 $k_{1}$ 和 Stephenson 的结果一致. $k_{7}$ 和 $k_{8}$ 误差较大, 这是 $\mathrm{CO}(v=7,8)$ 的布居很小, 拟合误差也较大造成的.

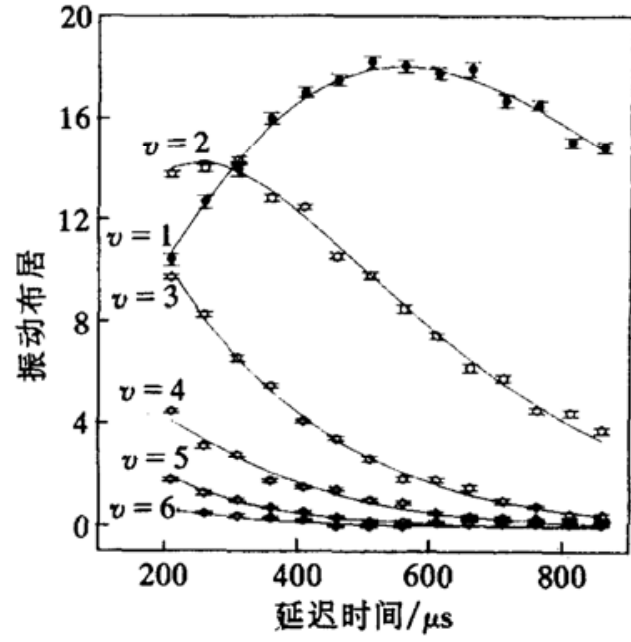

图 $3 \mathrm{CO}(v=1 \sim 6)$ 的振动布居及其随时间 的演变

实线为微分法计算得到的布居 
微分法计算布居如图 3 中实线所示, 与实验布居吻合很好.

表 $1 \mathrm{CO}(v)$ 与 $\mathrm{H}_{2} \mathrm{O}$ 的传能速率常数 (单位: $10^{-13} \mathrm{~cm}^{3} \cdot$ molecule $^{-1} \cdot \mathrm{s}^{-1}$ )

\begin{tabular}{|c|c|c|c|c|c|c|c|c|}
\hline$k_{v}$ & 1 & 2 & 3 & 4 & 5 & 6 & 7 & 8 \\
\hline 本 文 & $1.7 \pm 0.1$ & $3.4 \pm 0.2$ & $6.2 \pm 0.4$ & $8.0 \pm 1.0$ & $9.0 \pm 2.0$ & $12 \pm 3$ & $16 \pm 4$ & $18 \pm 7$ \\
\hline Stephenson ${ }^{[1]}$ & 2.0 & - & - & - & - & - & - & - \\
\hline
\end{tabular}

\section{3 讨论}

(1) 从传能速率常数来看, $\mathrm{CO}$ 向 $\mathrm{H}_{2} \mathrm{O}$ 的振动传能是很有效的, 要比 $\mathrm{CO}$ 向 $\mathrm{CO}_{2}$ 的振动传 能速率常数 (约 $10^{-14} \mathrm{~cm}^{3} \cdot$ molecule ${ }^{-1} \cdot \mathrm{s}^{-1}$ ) 高 1 2 个数量级. 这提醒人们要注意燃烧过程中 $\mathrm{H}_{2} \mathrm{O}$ 对 $\mathrm{CO}(v)$ 的重要猝灭作用. 随着振动量子数的增加, 速率常数单调增加. $1 \mathrm{~g} k_{v}$ 与 $1 \mathrm{~g} v$ 呈一直线关系, 斜率 $n \approx 1.0$, 符合经验关系 $k_{v}=v^{n} k_{1}{ }^{[8]}$, 如图 4 所示.

(2) 在 $\mathrm{H}_{2} \mathrm{O}$ 的 3 个振动模式中, 对称伸缩 $\left(\nu_{1}\right)$ 和反对称伸缩 $\left(\nu_{3}\right)$ 模与 $\mathrm{CO}(v)$ 之间的能级 差高达 $1500 \mathrm{~cm}^{-1}$ 以上, 因此难以传能. 实验中也没有观察到 $\mathrm{H}_{2} \mathrm{O}$ 的 $\nu_{1}$ 和 $\nu_{3}$ 的红外发射信 号. 只有 $(010)$ 与 $\mathrm{CO}(v \rightarrow v-1)$ 之间的能级差 $\Delta E$ 较小, 约 $500 \mathrm{~cm}^{-1}$, 可以发生传能. $\mathrm{SSH}$ 理论 ${ }^{[9]}$ 的估算结果见图 4. SSH 理论预测值与实验值的变化趋势一致, 但要比实验值低 3 4 倍. 高振动态偏差更大. 这种偏差可由两个原因造成. 一是由于 SSH 理论忽略了传能过程 中的 V-R 贡献, 而 $\mathrm{H}_{2} \mathrm{O}$ 的转动能级间隔大, 容易补偿振动传能的能量差; 二是 $\mathrm{CO}$ 与 $\mathrm{H}_{2} \mathrm{O}$ 可 能形成络合物. Yaron 等人 ${ }^{[10]}$ 已观测到 $\mathrm{OC}-\mathrm{H}_{2} \mathrm{O}$ 二聚体的微波谱. 在形成络合物时, $\mathrm{CO}$ 与 $\mathrm{H}_{2} \mathrm{O}$ 之间就可以发生多次“挤压”碰撞, 使得 $\mathrm{CO}(v)$ 的振动能充分传递 ${ }^{[11]}$.

（3）我们用量子化学从头算方法 $\left(\mathrm{HF} / 6-31 \mathrm{G} *\right.$ * ) 优化构型后, 得到两种不同的 $\mathrm{CO}-\mathrm{H}_{2} \mathrm{O}$ 的络合物, 如图 5 所示. 有趣的是, $\mathrm{C} \cdots \mathrm{H}-\mathrm{O}$ 型络合物的总能量比单体 $\left(\mathrm{CO}+\mathrm{H}_{2} \mathrm{O}\right)$ 低 $6.2 \mathrm{~kJ} /$ $\mathrm{mol}$, 比 $\mathrm{O} \cdots \mathrm{H}-\mathrm{O}$ 型络合物 (比单体低 $4.9 \mathrm{~kJ} / \mathrm{mol}$ ) 还要稳定. 实验上 ${ }^{[9]}$ 也只观察到了 $\mathrm{C} \cdots \mathrm{H}-\mathrm{O}$ 型络合物. 更深入的研究正在进行中.

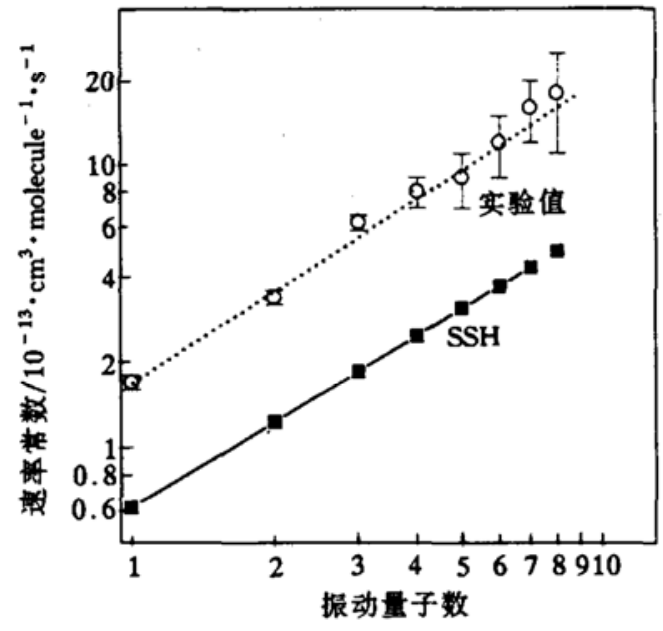

图 4 传能速率常数与振动量子数的关系 $\mathrm{SSH}$ 预测值与实验值的比较

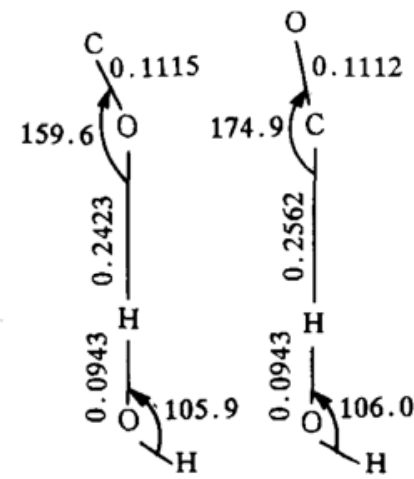

图 $5 \mathrm{CO}-\mathrm{H}_{2} \mathrm{O}$ 和 $\mathrm{OC}-\mathrm{H}_{2} \mathrm{O}$ 二聚体的结构示意图 键长单位: $\mathrm{nm}$, 键角单位: $\left({ }^{\circ}\right)$ 
致谢 本工作为国家自然科学基金(批准号:29773052)资助项目.

\title{
参考 文 献
}

1 Stephenson J C, Mosburg Jr E R. Vibrational energy transfer in CO from 100 to $300 \mathrm{~K}$. J Chem Phys, 1974, 60:3562

2 Buchwald $\mathrm{M} \mathrm{I}$, Bauer $\mathrm{S} \mathrm{H}$. Vibrational relaxation in $\mathrm{CO}_{2}$ with selected collision partners. $\mathrm{I}_{2} \mathrm{O}$ and $\mathrm{D}_{2} \mathrm{O}$. J Phys Chem, 1972, 76: 3108

3 Kurian J, Sreekanth A K. Laser schlieren study of vibrational relaxation of $\mathrm{N}_{2}$ by $\mathrm{H}_{2} \mathrm{O}$. Chem Phys, 1987, 114: 295

4 Shin H K. Importance of complex-mode collisions in the vibrational relaxation of strongly sttracting molecules. The $\mathrm{H}_{2} \mathrm{O}-\mathrm{HCl}$ system. Chem Phys Lett, 1993, $216(1,2): 27$

5 Lieb S G, Bevan J W. A traped collsion pair approach to vibrational predissociation in hydrogen-bonded complexes. Chem Phys Lett, 1985, 122(3): 284

6 王宝山, 顾月姝, 李 强, 等. 高振动激发态的 $V-V$ 传能研究一 I . $\mathrm{CO}(v)$ 向 $\mathrm{CO}_{2}$ 的振动传能,科学通报, 1998, 43 (22): $2399 \sim 2404$

7 Wang X B, Li H Z, Kong F A, et al. The vibrational quenching of $\mathrm{NO}(v=1 \sim 11)$ by $\mathrm{N}_{2} \mathrm{O}$ studied by time-resolved Fourier transform infrared emission spectroscopy. Chem Phys Lett, 1993, 208(3,4): 290

8 Bott J F. Vibrational relaxation of $\operatorname{DF}(v=1 \sim 4)$ in $\mathrm{D}_{2}, \mathrm{H}_{2}, \mathrm{~N}_{2}$, HF and $\mathrm{CO}_{2}$. J Chem Phys, 1979, 70(9): 4123

9 Schwartz R N, Slawsky Z I, Herzfeld K F. Calculation of vibrational relaxation times in gases. J Chem Phys, 1952, 20 : 1591

10 Yaron D, Peterson K I, Zolandz D. Water hydrogen bonding: The structure of the water-carbon monoxide complex. J Chem Phys, 1990, 92: 7095

11 Shin H K. Self-relaxation of vibrationally excited $\mathrm{H}_{2} \mathrm{O}$ molecules. J Chem Phys, 1993, 98(3): 1964

(1998-03-11 收稿)

\section{$\boldsymbol{\alpha}, \boldsymbol{\beta}$-不饱和酸铜 (II) 与腿三元配合物的研究}

\author{
王尧宇 (1) 时 茜 (2) 史启袩 (2) 高忆慈 (1) 周忠远 ${ }^{(3)}$
}

(1)兰州大学化学系, 兰州 730000; (2)西北大学化学系, 西安 710069; (3)中国科学院成都有机化学研究所, 成都 610041)

摘要 合成了丙烯酸铜 (II) 与腺和 $\alpha$-甲基丙烯酸铜 (II) 与胀形成的两种三元配合物, 通过元 素分析、IR 谱、热谱和磁化率等对配合物进行了表征。确定了配合物的组成为 $\mathrm{Cu}_{2} \mathrm{~A}_{4}\left(\left(\mathrm{NH}_{2}\right)_{2} \mathrm{CO}\right)_{2}\left(\mathrm{~A}=\mathrm{CH}_{2}=\mathrm{CHCOO}^{-}, \mathrm{CH}_{2}=\mathrm{C}\left(\mathrm{CH}_{3}\right) \mathrm{COO}^{-}\right)$, 提出了热分解机理, 测 定了 $\mathrm{Cu}_{2}\left(\mathrm{CH}_{2}=\mathrm{CHCOO}\right)_{4}\left(\left(\mathrm{NH}_{2}\right)_{2} \mathrm{CO}\right)_{2}$ 的晶体结构. 晶体属四方晶系, $\mathrm{P} 4_{2} / \mathrm{n}$ 群, 晶胞参 数: $a=b=1.23925(6), c=1.39805(16) \mathrm{nm} ; Z=4$. 配合物分子为 4 个羧基桥联双核 铜 (II) 的笼状结构, $\left(\mathrm{NH}_{2}\right)_{2} \mathrm{CO}$ 配体以 $\mathrm{O}$ 原子与 $\mathrm{Cu}$ (II) 配位, 在 $\mathrm{Cu}-\mathrm{Cu}$ 间有一对称中心, $\mathrm{Cu}-\mathrm{Cu}$ 键长为 $0.2609(4) \mathrm{nm}$.

\section{关键词 $\alpha, \beta$-不饱和酸铜（II）搌 三元配合物 热分解机理 晶体结构}

铜 (II) 的配合物有着丰富多样的立体结构, 它与有机酸形成的配合物还广泛存在于生命 体系中, 对生命体系有着特殊的生物活性和催化作用, 因而, 近年来这一领域的研究倍受关 